Michał Kasprzak ${ }^{1}$, Małgorzata Molska1, Karolina Obońska', Emilia Kolasińska', Julia Maria Kubica1, Adam Arndt ${ }^{1}$, Ewa Laskowska ${ }^{2}$, Ewa Obońska ${ }^{3}$, Marlena Ewertowska ${ }^{1}$, Joanna Sikora ${ }^{3}$, Alicja Janicka ${ }^{1}$, Tomasz Fabiszak ${ }^{1}$, Grzegorz Grześk ${ }^{3}$, Marek Koziński², Jacek Kubica ${ }^{1}$

${ }^{1}$ Department of Cardiology and Internal Medicine

2Department of Principles of Clinical Medicine

3Department of Pharmacology and Therapy

Nicolaus Copernicus University, Collegium Medicum in Bydgoszcz, Poland

\title{
Variability of prasugrel antiplatelet effect in patients with acute coronary syndrome
}

\author{
Corresponding author: \\ Michał Kasprzak, MD, PhD \\ Department of Cardiology \\ and Internal Medicine, \\ Collegium Medicum, \\ Nicolaus Copernicus University \\ 9 Skłodowskiej-Curie Street \\ 85-094 Bydgoszcz, Poland \\ Phone: +48525854023 \\ Fax: +48525854024 \\ E-mail: medkas@o2.pl
}

Folia Medica Copernicana 2015; Volume 3, Number 3, 117-124 10.5603/FMC.2015.0006 Copyright (C) 2015 Via Medica ISSN 2300-5432

\begin{abstract}
Background. Many reports have demonstrated excessive variability in response to clopidogrel, the most commonly used P2Y12 receptor antagonist. Clopidogrel resistant patients are at increased risk of cardiovascular (CV) events. Prasugrel is a new P2Y12 inhibitor that provides greater and faster platelet inhibition and reduces $\mathrm{CV}$ events more effectively than clopidogrel. The aim of this study was to evaluate the variability and efficacy of prasugrel antiplatelet activity in patients presenting with acute coronary syndrome (ACS). Materials and methods. The study was designed as a prospective, single-center, non-randomized, observational trial. Platelet reactivity (PR) was assessed with the VeryfyNow assay three times during hospitalization in forty-two patients undergoing percutaneous coronary intervention (PCI) for ACS and treated with standard doses of prasugrel.

Results. Platelet aggregation with prasugrel displayed relatively high variability. The platelet aggregation was lowest on the $3^{\text {rd }}$ day of the treatment at 4 p.m. and was significantly different from the measurements obtained on the $3^{\text {rd }}$ and $4^{\text {th }}$ day in the morning (6.0 v. $8.5 \mathrm{U} ; p=0.0005$ and 6.0 v. $36.5 \mathrm{U} ; \mathrm{p}<0.00001$, respectively), with the latter two differing significantly from each other $(p=0.002)$. All participants were successfully treated with prasugrel achieving PR < 208 PRU in each measurement, whereas 42.9-80.9\% (depending on sampling point) of patients presented very low platelet activity. The subgroups of stable and persistent low PR included a higher percentage of active smokers (73.3 v. 40.7\%; $p=0.04$ and 80.0 v. 43.8\%; $p=0.04$, respectively).

Conclusions. Prasugrel treatment is associated with high variability of PR. Nonetheless, prasugrel is a highly effective antiplatelet drug. Active smoking may predispose to strong and stable on-prasugrel platelet inhibition. Key words: prasugrel, clopidogrel, platelet reactivity, variability, acute coronary syndrome
\end{abstract}

Folia Medica Copernicana 2015; 3 (3): 117-124

\section{Introduction}

Platelet activation and aggregation play a pivotal role in the pathophysiology of acute coronary syndromes (ACS) [1]. Dual antiplatelet therapy (DAT) with aspirin and one of the platelet P2Y12 receptor antagonists reduces the risk of thrombotic events. This treatment is recommended in the management of patients with ACS and in patients after stent implantation [2-4]. Many reports have demonstrated excessive variability in response to clopidogrel, the most commonly used P2Y12 receptor antagonist, with a substantial rate of high on-treatment platelet reactivity (HTPR) [5-8]. Subjects with HTPR undergoing percutaneous coronary intervention $(\mathrm{PCl})$ were shown to be at increased risk of cardiovascular (CV) events [6, 9]. Prasugrel is a new P2Y12 inhibitor that provides greater and faster platelet inhibition. It was shown to reduce $\mathrm{CV}$ events more effectively than clopidogrel in ACS patients undergoing $\mathrm{PCl}$ [10-12].

The aim of this study was to evaluate the inter-individual and intra-individual variability of prasugrel antiplatelet activity in patients with ACS treated with $\mathrm{PCl}$. 


\section{Materials and methods}

\section{Study design and population}

This study was designed as a prospective, single-center, non-randomized, observational trial. Forty-two consecutive patients admitted to the Department of Cardiology and Internal Medicine, Collegium Medicum, Nicolaus Copernicus University, Bydgoszcz and treated with $\mathrm{PCl}$ for ACS were prospectively recruited into the study between 24 July 2013 and 16 May 2014. The enrollment during first two days of hospitalization.

Study exclusion criteria included:

- age under 18 or over 75 years;

- history of ischemic stroke or transient ischemic attack;

- body weight below 60 kilograms;

- severe liver failure (Child-Pugh class C);

- need for prolonged use of oral anticoagulant therapy, heparin or fondaparinux;

- bleeding disorders (including thrombocytopenia $\left.<100 \times 10^{3} / \mu \mathrm{L}\right)$;

- anemia (defined as hemoglobin concentration $<10.0 \mathrm{~g} / \mathrm{dL})$;

- active inflammation;

- cardiogenic shock on admission;

- heart failure class III and IV according to the New York Heart Association (NYHA) classification;

- any contraindication to prasugrel due to increased risk of bleeding according to the attending physician. All participants provided written informed consent. The study was approved by the Ethical Committee of the Nicolaus Copernicus University in accordance with the Declaration of Helsinki.

\section{Pharmacotherapy}

Directly after enrollment for the study patients were treated with a loading dose $(60 \mathrm{mg})$ of prasugrel regardless of earlier antiplatelet treatment. The day of study inclusion was marked as "day 0". Throughout the following hospitalization period prasugrel was continued in a single daily dose of $10 \mathrm{mg}$ administered at 8.00 a.m. Concomitant medication in all patients included aspirin (300 mg loading dose given immediately after establishing ACS diagnosis, followed by a $75 \mathrm{mg}$ maintenance dose once daily), ramipril and bisoprolol in doses adjusted for resting heart rate and blood pressure, and atorvastatin $(40 \mathrm{mg})$. All medications were administered at 8.00 a.m., except for atorvastatin, which was administered at 8.00 p.m. Gastroprotection with pantoprazole was given at the discretion of the attending physician.
Measurement of platelet aggregation

Blood samples for platelet function testing were collected at 3 time points: on the $3^{\text {rd }}$ day of the maintenance treatment with $10 \mathrm{mg}$ daily prasugrel dose (called "day 3 ") at 8:00 a.m. (before prasugrel administration), on "day 3 " at 4:00 p.m. (8 h after the previous dose of prasugrel) and on the $4^{\text {th }}$ day (called "day 4") at 8:00 a.m. (prior to prasugrel administration). Prasugrel dosing regimen and timing of platelet testing are presented in Table 1.

Blood samples for platelet reactivity (PR) assessment were drawn through a short venous catheter inserted into a forearm vein. Platelet function testing was performed with the VeryfyNow (Accumetrics Inc, San Diego, CA) point-of-care P2Y12 function assay. The results are reported in $\mathrm{P} 2 \mathrm{Y} 12$ reaction unit (PRU). According to the previous studies, PRU $>208$ was considered HTPR associated with an increased risk of thrombotic events [13, 14], while PRU $<30$ PRU was considered LTPR (low on-treatment platelet reactivity) associated with an increased risk of bleeding complications [14-16].

\section{Statistical analysis}

Due to non-normal distribution of the investigated continuous variables as assessed with the Shapiro-Wilk test, continuous variables are reported as median values and their interquartile ranges. Intergroup comparisons were performed with the Mann-Whitney unpaired rank sum test, whereas the Wilcoxon matched-pairs rank sum test was applied for comparisons within the groups. Independent categorical variables were compared using the $\chi^{2}$ test with the Yates' correction if required. Multiple comparisons were analyzed with the ANOVA Friedman test. Values of two sided $p<0.05$ were regarded as statistically significant;

Table 1. Prasugrel dosing regimen and measurements of platelet activity

\begin{tabular}{lll}
\hline Day & Hour & Action taken \\
\hline "Day 0" & & Administration of $60 \mathrm{mg}$ of prasugrel \\
"Day 1" & 8:00 a.m. & Administration of $10 \mathrm{mg}$ of prasugrel \\
"Day 2" & 8:00 a.m. & Administration of $10 \mathrm{mg}$ of prasugrel \\
"Day 3" & 8:00 a.m. & Administration of $10 \mathrm{mg}$ of prasugrel \\
& 8:00 a.m. & Measurement of platelet reactivity \\
& 4:00 p.m. & Measurement of platelet reactivity \\
"Day 4" & 8:00 a.m. & Administration of 10 mg of prasugrel \\
& 8:00 a.m. & Measurement of platelet reactivity \\
\hline
\end{tabular}


$0.05 \leq \mathrm{p}<0.1$ was considered a trend towards statistical significance. The statistical analysis was carried out using the Statistica 10.0 statistical software (StatSoft, Tulsa, USA).

\section{Results}

The baseline characteristics of the study population are presented in Table 2. We observed high inter-individual and intra-individual variability of platelet reactivity on prasugrel. Inter-individual variability, especially at the morning sampling points, is well reflected by broad interquartile (day 3: 4.0-40.0; day 4: 6.0-55.0) and non-outlier ranges (day 3: 0.0-93.0; day $4: 1.0-127.0)$ (Fig. 1). In contrast, in the afternoon the dispersion of the results of platelet reactivity assessment markedly decreased (3.0-14.0 and 0.0-25.0, respectively).

High intra-individual variability was reflected by huge differences in the platelet reactivity measure-

Table 2. Baseline characteristics of the study population $(n=42)$. Continuous variables are presented as median (lower quartile-upper quartile) while categorical variables are presented as numbers (percent)

\begin{tabular}{|c|c|}
\hline Variable & Value \\
\hline Age (years) & $56.0(52.0-62.0)$ \\
\hline Gender (male/female) & $38(90.5 \%) / 4(9.5 \%)$ \\
\hline \multicolumn{2}{|l|}{ Final diagnosis } \\
\hline NSTEMI/UA & $12(28.6 \%)$ \\
\hline STEMI & $30(71.4 \%)$ \\
\hline Hypertension & $23(54.8 \%)$ \\
\hline Diabetes mellitus & $11(26.2 \%)$ \\
\hline Current smoker & $22(52.4 \%)$ \\
\hline LVEF (\%) & $45.0(38.0-50.0)$ \\
\hline \multicolumn{2}{|l|}{ Lipid profile } \\
\hline Total cholesterol [mg/dL] & $224.0(190.0-244.0)$ \\
\hline LDL cholesterol [mg/dL] & $152.0(119.0-174.0)$ \\
\hline HDL cholesterol [mg/dL] & $50.0(39.5-56.5)$ \\
\hline Triglycerides [mg/dL] & $95.0(69.0-145.0)$ \\
\hline $\mathrm{PLT}\left[10^{3} / \mu \mathrm{L}\right]$ & 201.5 (173.0-243.0) \\
\hline MPV [fL] & $11.4(10.6-11.8)$ \\
\hline $\begin{array}{l}\text { Proton pump inhibitor } \\
\text { administration }\end{array}$ & $12(28.6 \%)$ \\
\hline GFR $\left[\mathrm{ml} / \mathrm{min} / 1.73 \mathrm{~m}^{2}\right]$ & $99.5(90.5-108.5)$ \\
\hline \multicolumn{2}{|c|}{$\begin{array}{l}\text { GFR - glomerular filtration rate; HDL - high-density lipoprotein; LDL } \\
\text { - low-density lipoprotein; LVEF - left ventricular ejection fraction; } \\
\text { MPV - mean platelet volume; NSTEMI - non ST-segment elevation } \\
\text { myocardial infarction; PLT - platelets; STEMI - ST-segment elevation } \\
\text { myocardial infarction; UA - unstable angina }\end{array}$} \\
\hline
\end{tabular}

ments obtained at different sampling points. The lowest platelet reactivity was observed on day 3 at 4:00 p.m. and differed significantly as compared with reactivity measured on day 3 at 8:00 a.m. (6.0 [3.0-14.0] v. 8.5 [4.0-40.0] $U ; p=0.0005)$, and on day 4 at 8:00 a.m. (6.0 [3.0-14.0] v. 36.5 [6.0-55.0] U; $\mathrm{p}<0.00001)$. There was also a significant day-today difference in the morning platelet reactivity. The morning platelet reactivity was higher on day 4 than on day 3 (36.5 [6.0-55.0] v. 8.5 [4.0-40.0] U; $p=0.002)$. The ANOVA Friedman test confirmed a significant heterogeneity in the platelet reactivity assessed at different sampling points ( $p<0.00001$; Fig. 1). Intra-individual platelet aggregation variability is also well depicted when the measurements of individual patients are followed (Fig. 2).

All study participants were effectively treated with prasugrel during hospitalization (PRU $<208$ at each measurement point). LTPR was observed in 77 out of 126 measurements $(61.1 \%)$. The highest prevalence of LTPR was found on day 3 at 4:00 p.m. (80.9\%), while the lowest prevalence of LTPR was seen on day 4 at 8.00 a.m. (42.9\%) (Fig. 3). In 15 subjects (35.7\%) LTPR was observed at all three measurement points. When this subset of patients was compared with subjects who had at least one platelet aggregation measurement within therapeutic range, a statistically higher percentage of active smokers was found in the persistent LTPR group (73.3 v. 40.7\%; 0.04). There were also tendencies for lower age (54.0 [51.0-58.0] v. 59.0 [53.0-66.0] years; $p=0.051$ ) and higher high-density lipoprotein (HDL) cholesterol concen-

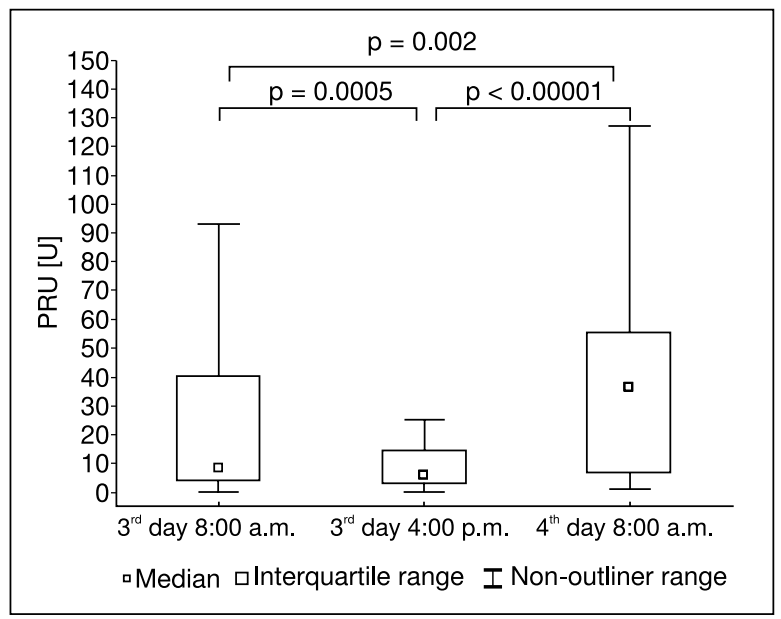

Figure 1. Diurnal and day-to-day variation of platelet aggregation as assessed with the Veryfy Now P2Y12 assay. Statistical significance $p$ values for comparisons of two sampling points are presented on the figure. Statistical significance for inter-measurement heterogeneity at three time points is $<0.00001$ 


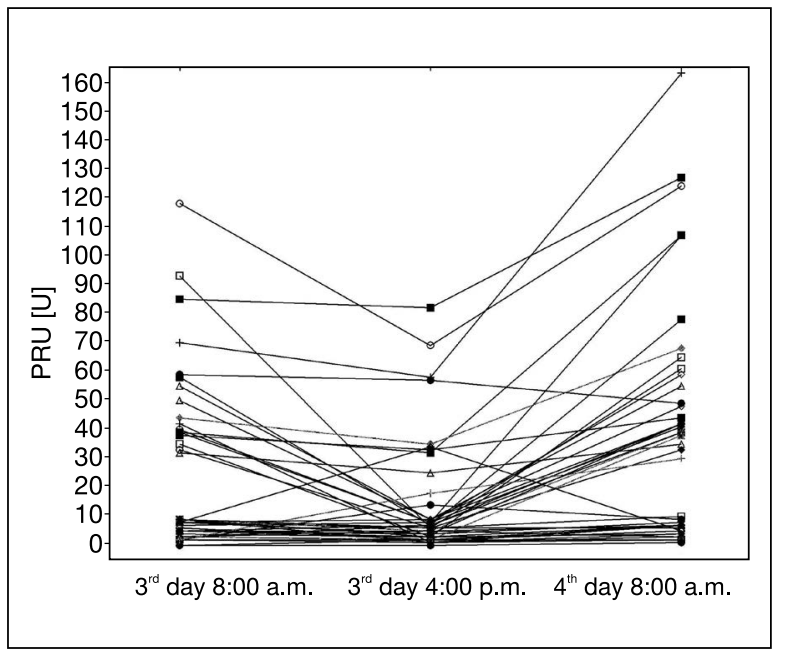

Figure 2. Diurnal and day-to-day platelet aggregation variation of individual patients as assessed by the VeryfyNow P2Y12 assay

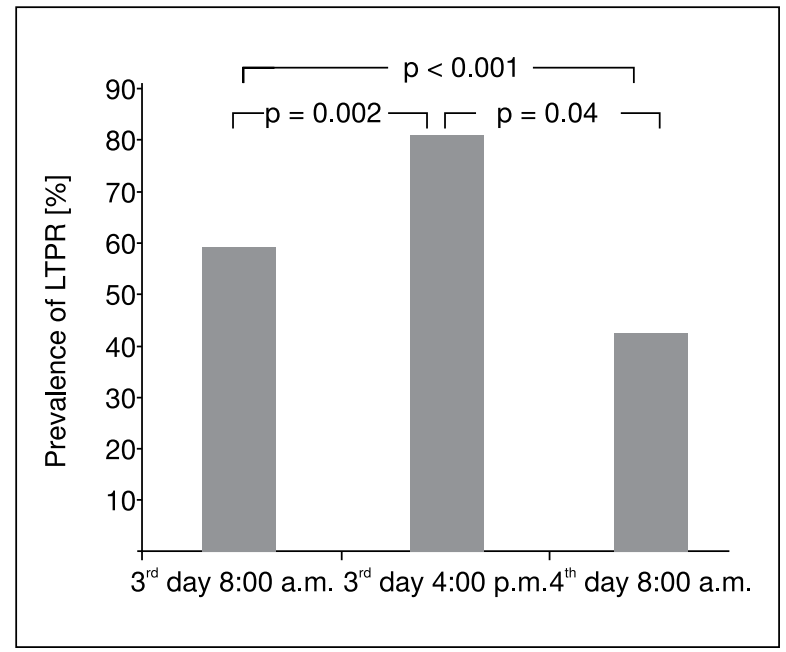

Figure 3. Prevalence of low on-treatment platelet reactivity (LTPR) at each sampling point

Table 3. Comparison of characteristics of patients with and without persistent low on-treatment platelet reactivity. Continuous variables are presented as median (lower quartile-upper quartile) while categorical variables are presented as numbers (percent)

\begin{tabular}{|c|c|c|c|}
\hline Variable & $\begin{array}{l}\text { LTPR in all three measurements } \\
\qquad(n=15)\end{array}$ & $\begin{array}{l}\text { At least one measurement of PR } \\
\text { within therapeutic range }(n=37)\end{array}$ & $\mathbf{p}$ \\
\hline Age (years) & $54.0(51.0-58.0)$ & $59.0(53.0-66.0)$ & 0.051 \\
\hline Gender (male/female) & $15(100.0 \%) / 0(0.0 \%)$ & $23(85.2 \%) / 4(14.8 \%)$ & 0.31 \\
\hline \multicolumn{4}{|l|}{ Final diagnosis } \\
\hline NSTEMI/UA & $4(26.7 \%)$ & $8(29.6 \%)$ & 0.83 \\
\hline STEMI & $11(73.3 \%)$ & 19 (70.4\%) & \\
\hline Hypertension & $7(46.7 \%)$ & $16(59.3 \%)$ & 0.43 \\
\hline Diabetes mellitus & $3(20.0 \%)$ & $8(29.6 \%)$ & 0.75 \\
\hline Current smoker & $11(73.3 \%)$ & $11(40.7 \%)$ & 0.04 \\
\hline LVEF (\%) & $45.0(40.0-50.0)$ & $40.0(38.0-50.0)$ & 0.51 \\
\hline \multicolumn{4}{|l|}{ Lipid profile } \\
\hline Total cholesterol [mg/dL] & $241.0(191.0-263.0)$ & $218.0(187.0-236.0)$ & 0.22 \\
\hline LDL cholesterol [mg/dL] & $152.0(119.0-176.0)$ & $153.0(123.0-172.0)$ & 0.99 \\
\hline HDL cholesterol [mg/dL] & $52.5(47.0-58.0)$ & $46.5(36.0-55.0)$ & 0.07 \\
\hline Triglycerides [mg/dL] & $115.5(82.0-152.0)$ & $93.0(69.0-143.0)$ & 0.44 \\
\hline PLT $\left[10^{3} / \mu \mathrm{L}\right]$ & $214.0(188.0-243.0)$ & $191.5(169.0-250.0)$ & 0.58 \\
\hline MPV [fL] & $11.4(10.3-12.1)$ & $11.4(10.7-11.8)$ & 1.00 \\
\hline Proton pump inhibitor administration & $5(33.3 \%)$ & $7(25.9 \%)$ & 0.88 \\
\hline GFR $\left[\mathrm{ml} / \mathrm{min} / 1.73 \mathrm{~m}^{2}\right]$ & $100.0(96.0-114.0)$ & $99.0(89.0-108.0)$ & 0.40 \\
\hline
\end{tabular}


Table 4. Comparison of characteristics of patients with and without stable platelet reactivity. Continuous variables are presented as median (lower quantile-upper quantile) while categorical variables are presented as numbers (percent)

\begin{tabular}{|c|c|c|c|}
\hline Variable & PR fluctuations $<5$ PRU $(n=10)$ & PR fluctuations $\geq 5$ PRU $(n=32)$ & $\mathbf{p}$ \\
\hline Age (years) & $54.5(51.0-58.0)$ & $56.5(52.5-64.5)$ & 0.23 \\
\hline Gender (male/female) & $10(100.0 \%) / 0(0.0 \%)$ & $28(87.5 \%) / 4(12.5 \%)$ & 0.58 \\
\hline \multicolumn{4}{|l|}{ Final diagnosis } \\
\hline NSTEMI/UA & $2(20.0 \%)$ & $10(31.3 \%)$ & 0.77 \\
\hline STEMI & $8(80.0 \%)$ & $22(68.7 \%)$ & \\
\hline Hypertension & $3(30.0 \%)$ & $20(62.5 \%)$ & 0.07 \\
\hline Diabetes mellitus & $0(0.0 \%)$ & $11(34.4 \%)$ & 0.08 \\
\hline Current smoker & $8(80.0 \%)$ & $14(43.8 \%)$ & 0.04 \\
\hline LVEF (\%) & $45.0(40.0-50.0)$ & $42.5(36.5-50.0)$ & 0.39 \\
\hline \multicolumn{4}{|l|}{ Lipid profile } \\
\hline Total cholesterol [mg/dL] & $232.0(192.0-263.0)$ & $218.0(187.0-236.0)$ & 0.32 \\
\hline LDL cholesterol [mg/dL] & $152.0(123.0-176.0)$ & $152.5(112.0-174.0)$ & 0.89 \\
\hline HDL cholesterol [mg/dL] & $52.5(48.0-53.0)$ & $47.0(37.0-57.0)$ & 0.22 \\
\hline Triglycerides [mg/dL] & $110.0(82.0-133.0)$ & $194.0(172.5-244.5)$ & 0.71 \\
\hline $\mathrm{PLT}\left[10^{3} / \mu \mathrm{L}\right]$ & $214.0(188.0-242.0)$ & $194.0(172.5-244.5)$ & 0.76 \\
\hline MPV [fL] & $11.3(10.6-12.2)$ & $11.4(10.7-11.6)$ & 0.60 \\
\hline $\begin{array}{l}\text { Proton pump inhibitor } \\
\text { administration }\end{array}$ & $3(30.0 \%)$ & $9(28.1 \%)$ & 0.77 \\
\hline GFR $\left[\mathrm{ml} / \mathrm{min} / 1.73 \mathrm{~m}^{2}\right]$ & $100.5(96.0-114.0)$ & $97.5(89.0-108.0)$ & 0.30 \\
\hline
\end{tabular}

GFR — glomerular filtration rate; HDL — high-density lipoprotein; LDL — low-density lipoprotein; LVEF — left ventricular ejection fraction; MPV - mean platelet volume; NSTEMI — non ST-segment elevation myocardial infarction; PLT — platelets; PR — platelet reactivity; STEMI - ST-segment elevation myocardial infarction; UA — unstable angina

tration (52.5 [47.0-58.0] v. 46.5 [36.0-55.0] mg/dL; $p=0.07$ ) in this group (Tab. 3). Interestingly, the persistent LTPR subset of patients comprised only men, while all 4 women participating in the study had at least one platelet reactivity measurement within normal range. This difference however was not statistically significant, which may be due to the small representation of women in our study.

Despite generally high variability in diurnal and dayto-day variations in platelet reactivity observed in our study we found 10 patients (23.8\%) with fluctuations in VeryfyNow P2Y12 assay results below 5 PRU. Patients with the stable platelet function measurements significantly more often were active smokers (80.0 v. $43.8 \% ; p=0.04$ ) and displayed tendencies for lower prevalence of hypertension (30.0 v. $62.5 \%$; $p=0.07$ ) and diabetes (0.0 v. 34.4\%; $p=0.08$ ) (Tab. 4). All 4 women were in the higher PR fluctuation group, however this difference in gender distribution was statistically insignificant.

Platelet reactivity assessed with the VeryfyNow P2Y12 assay at each sampling point was unrelated to platelet count or mean platelet volume.

\section{Discussion}

Efficient and stable platelet inhibition is the cornerstone of the treatment for patients with ACS and/or undergoing PCl. Prasugrel provides a more potent and stable antiplatelet effect when compared with clopidogrel $[17,18]$. However, the main finding of our study is the relatively high inter-individual and intra-individual variability of platelet reactivity as assessed with the VeryfyNow P2Y12 assay among patients treated with a maintenance dose of prasugrel in the stable phase of ACS (Fig. 1, Fig. 2). Taking into account the relatively small population included in our study, significant differences of diurnal and day-to-day aggregation proved the existence of substantial variability. However, the analysis of data presented in Figure 2 leads to a conclusion that even with the prominent intra-individual differences in aggregation there is a subgroup of patients presenting a stable and relatively low level of platelet reactivity. Further analysis revealed that 10 patients with low PR fluctuations, below 5 PRU, more often were active smokers. Moreover, those patients tended to have a lower prevalence of diabetes mellitus and hypertension (Tab. 4). 
Diabetes mellitus is associated with a chronic inflammatory state promoting platelets hyperactivity $[19,20]$. Several studies showed resistance to a conventional antiplatelet therapy with aspirin and clopidogrel in diabetic patients [2, 20]. Prasugrel has been proven to be more effective than clopidogrel in both diabetic and non-diabetic subjects [21]. This pharmacodynamic effectiveness translated into a better clinical outcome in patients treated with prasugrel compared with clopidogrel in the TRITON-TIMI 38 study [10]. The composite ischemic event rate with prasugrel was diminished by $14 \%$ in non-diabetic patients, by $26 \%$ in non-insulin-treated diabetics, and by $37 \%$ in those treated with insulin [22].

Despite superior antiplatelet potency of prasugrel its effect remains suboptimal in some subsets of patient. Diabetes is one of the strongest factors of high on-prasugrel platelet reactivity $[23,24]$. Interestingly, in our population none of 11 diabetic patients displayed low PRU fluctuation. This suggests that diabetes is a risk factor not only for HTPR, but also for higher intra-individual variability.

Hypertension was also identified as a risk factor of aspirin and clopidogrel resistance in some studies [25, 26]. Recently, Verdoia et al. showed that hypertension may also be a risk factor for ticagrelor non-responsiveness. Among other risk factors, hypertension was significantly associated with high platelet reactivity in the univariate analysis. However, age $\geq 70$ years, concomitant therapy with beta-blockers and platelets count were the only independent predictors of high on-ticagrelor platelet reactivity in the multivariate model [27]. Also retrospective analysis conducted by Bae et al. and a recent registry of $\mathrm{PCl}$-treated patients did not indicate hypertension as a factor of high on-prasugrel platelet reactivity $[23,24]$. In our study, a tendency for lower hypertension prevalence in the stable PR subset of patients was revealed, suggesting that hypertension might cause some variability in antiplatelet response.

It is a well-known fact that antiplatelet efficacy of clopidogrel is higher in smokers than in non-smokers. In a study conducted in 377 patients with coronary artery disease who had undergone $\mathrm{PCl}$ and were treated with clopidogrel, significantly lower PR was found in smokers. An association between smoking and antiplatelet activity was also confirmed with the multivariate analysis [28]. Moreover, the clinical benefit of clopidogrel in preventing cardiovascular events was greater in smokers than in non-smokers, a phenomenon termed the "smoker's paradox" $[29,30]$. The explanation for this observation is cytochrome P 450 (CYP) 1A2 and 2B6 induction by cigarette smoking which results in a greater active metabolite generation [31]. Prasugrel undergoes less complex metabolization without involving CYP1A2 and therefore its efficacy should not be influenced by the smoking status [32]. However, a pharmacokinetic and pharmacodynamic study conducted by Gurbel et al. showed slightly stronger platelet inhibition by prasugrel in smokers than non-smokers. This study also confirmed stronger antiplatelet potency of clopidogrel amongst smokers [32]. Our results suggest that the antiplatelet effect of prasugrel is more stable in smokers.

Although the subgroup of low PR fluctuation in our study consisted exclusively of men, this may be by chance, since our study population comprised only 4 women. To draw any conclusions regarding the platelet response variability in relation to gender, a study with an adequate number of female participants should be conducted.

We observed PR less than 208 PRU in every platelet measurement performed during the study. The treatment with clopidogrel, a still widely used second generation P2Y12 receptor blocker, results in an inadequate platelet inhibition in approximately one-third of patients with ACS [33]. Newer third generation P2Y12 antagonists, such as prasugrel, showed more effective platelet inhibition [12, 34, 35]. In a study by Alexopoulos et al., 27 STEMI patients treated with prasugrel presented 11.5\% HTPR $6 \mathrm{~h}$ after loading dose administration and $0 \% 5$ days after the treatment initiation, as assessed with the VeryfyNow P2Y12 assay [36]. Similarly, in a study conducted by Laine et al. with 44 STEMI patients treated with prasugrel, HTPR measured 6-12 $\mathrm{h}$ after a loading dose of prasugrel and defined as VASP index above or equal to $50 \%$, was reported in 4 patients $(9,1 \%)$ [37]. Prasugrel was also shown to be effective in 44 ACS patients exhibiting HTPR following a clopidogrel loading dose. The rate of HTPR after 15 days of the maintenance treatment with prasugrel was only $2.4 \%$ in this high risk population [38].

On the other hand, up to $80.9 \%$ of patients enrolled into our study presented PR $<30$ PRU, a level which has been shown to increase the risk of bleeding complications [14-16]. Our results are consistent with the study conducted by Laine et al., with the latter showing a $63.6 \%$ incidence of LTPR (defined as VASP index $\leq 16 \%$ ) measured 6 to $12 \mathrm{~h}$ after prasugrel loading dose administration [37]. A lower prevalence of LTPR (using the same VASP index $\leq 16 \%$ criterion), equal to $27.9 \%$ of patients, was reported by Bonello et al., with a concomitant 4.7-fold higher rate of bleeding events during a 1-year follow-up (15.6\% v. 3.3\%) [15]. In another study utilizing the VeryfyNow P2Y12 assay and the same cut-off point as in our study ( $<30 \mathrm{PRU}$ ), the LTPR rate after 15 days of prasugrel treatment was $45.6 \%$ [39].

More than one-third of our population had persistent LTPR (PR < 30 PRU in all three measurements). We believe that this subgroup of patients is of remarkably high risk of bleeding complications. Identifying such 
individuals and switching to less potent clopidogrel, as proposed by Kerneis et al., might be a reasonable option. However, this approach needs to be validated in large clinical trials as it may lead to ischemic events in clopidogrel non-responders [39].

In our study, patients with persistent LTPR more often were smokers which seems to confirm the stable and high antiplatelet potency of prasugrel in tobacco users as discussed above. As expected, patients from this group tended to be younger, as advanced age is a known risk factor of HTPR $[8,27]$.

Interestingly, patients with persistent LTPR presented a tendency for higher HDL cholesterol concentrations. Similar results regarding the association between clopidogrel resistance and lower HDL concentrations were reported for Jordanian and Serbian populations [26, 40].

\section{Limitations}

Several limitations of our study should be acknowledged. Firstly, only one method of platelet function assessment was applied. Secondly, the study population size was small. Thirdly, the spectrum of data acquired by the researchers did not include some variables bearing potential impact on on-treatment platelet reactivity (e.g. inflammatory markers).

\section{Conclusions}

Our study showed relatively high inter- and intra-individual variability of platelet reactivity among patients in the stable phase of ACS, treated with a maintenance dose of prasugrel. Additionally, we pointed out high antiplatelet effectiveness of prasugrel, as all PR measurements were below the threshold indicating high thrombotic risk and two-thirds of the PR measurements were below the threshold of high bleeding risk. Active smoking might predispose to strong and stable on-prasugrel platelet inhibition.

\section{References}

1. Kubica J, Adamski P, Ostrowska M et al. Influence of Morphine on Pharmacokinetics and Pharmacodynamics of Ticagrelor in Patients with Acute Myocardial Infarction (IMPRESSION): study protocol for a randomized controlled trial. Trials 2015; 16:198

2. Kubica A, Obońska K, Kasprzak M et al. Prediction of high risk of non-adherence to antiplatelet treatment. Kardiol Pol 2015; 23 [Epub ahead of print]

3. Windecker S, Kohl P, Alfonso F et al. 2014 ESC/EACTS Guidelines on myocardial revascularization. Eur Heart J 2014: 35: 2541-2619.

4. Kubica J. The optimal antiplatelet treatment in emergency setting. Folia Medica Copernicana 2014; 2: 73-76

5. Crescente M, Di Castelnuovo A, lacoviello L, Vermylen J, Cerletti C de Gaetano $G$. Response variability to aspirin as assessed by the platelet function analyzer (PFA)-100. A systematic review. Thromb Haemost 2008; 99: 14-26.
6. Kozinski M, Bielis L, Wisniewska-Szmyt $\mathrm{J}$ et al. Diurnal variation in platelet inhibition by clopidogrel. Platelets 2011; 22: 579-587.

7. Kubica A, Obońska K, Kasprzak M et al. The impact of metabolic syndrome on the antiplatelet effect of clopidogrel and aspirin in patients with acute coronary syndrome. Folia Med Copernicana, 2014; 2: 66-72.

8. Kubica A, Kasprzak M, Siller-Matula $J$ et al. Time-related changes in determinants of antiplatelet effect of clopidogrel in patients after myocardial infarction. Eur J Pharmacol 2014; 5: 47-54.

9. Eikelboom JW, Hankey GJ, Thom J et al. Clopidogrel for high atherothrombotic risk and ischemic stabilization, management and avoidance (CHARISMA) investigators. Incomplete inhibition of thromboxane biosynthesis by acetylsalicylic acid: Determinants and effect on cardiovascular risk. Circulation 2008; 118: 1705-1712.

10. Wivott SD, Braunwald E, MCCabe $\mathrm{CH}$ et al. Prasugrel versus clopidogrel in patients with acute coronary syndromes. N Engl J Med 2007; 357: 2001-2015.

11. Navarese, EP, Verdoia M, Schaffer $A$ et al. Iscaemic and bleeding comparisons with new, compared to standard, ADP antagonist regiments in acute coronary syndromes: a meta-analysis of randomized trials. QJM 201; 104: 561-569.

12. T. Jernberg, C.D. Payne, K.J. Winters et al. Prasugrel achieves greater inhibition of platelet aggregation and a lower rate of non-responders compared with clopidogrel in aspirin-treated patients with stable coronary artery disease. Eur Heart J 2006; 27: 1166-1173.

13. Price MJ, Angiolillo DJ, Teirstein PS et al. Platelet reactivity and cardiovascular outcomes after percutaneous coronary intervention: a time-dependent analysis of the Gauging Responsiveness with VerifyNow P2Y12 assay: Impact on Thrombosis and Safety (GRAVITAS) trial. Circulation 2011; 124: 1132-1137.

14. Tantry US, Bonello L, Arandi et al. Consensus and future directions on the definition of high on-treatment platelet reactivity to adenosine diphosphate. J Am Coll Cardiol, 2013; 62: 2261-2273.

15. Bonello L, Mancini J, Pansieri M et al. Relationship between post-treatment platelet reactivity and ischemic and bleeding events at 1 -year follow-up in patients receiving prasugrel. J Thromb Haemost 2012; 10: 1999-2005.

16. Ferreiro JL, Sibbing D, Angiolillo DJ. Platelet function testing and risk of bleeding complications. Thromb Haemost 2010; 103: 1128-1135.

17. Schrör K, Siller-Matula JM, Huber K. Pharmacokinetic basis of the antiplatelet action of prasugrel. Fundamental and Clinical Pharmacology 2012; 26: 39-46.

18. Payne CD, Li YG, Small DS et al. Increased active metabolite formation explains the greater platelet inhibition with prasugrel compared to high-dose clopidogrel. J Cardiovasc Pharmacol 2007; 50: 555-562.

19. Hillegass WB, Brott BC, Dobbs JC, Papapietro SE, Misra VK, Zoghbi GJ. Oral antiplatelet therapy in diabetes mellitus and the role of prasugrel: an overview. Vasc Health Risk Manag 2011; 7: 445-459.

20. Serebruany VL, Malinin A, Ong S, Atar D. Patients with metabolic syndrome exhibit higher platelet activity than those with conventional risk factors for vascular disease. J Thromb Thrombolysis 2008; 25: 207-213.

21. Wiviott SD, Trenk D, Frelinger AL et al. Prasugrel compared with high loading- and maintenance-dose clopidogrel in patients with planned percutaneous coronary intervention: The Prasugrel in Comparison to Clopidogrel for Inhibition of Platelet Activation and Aggregation-Thrombolysis in Myocardial Infarction 44 trial. Circulation 2007; 116: 2923-2932.

22. Wiviott SD, Braunwald E, Angiolillo DJ et al. Greater clinical benefit of more intensive oral antiplatelet therapy with prasugrel in patients with diabetes mellitus in the trial to assess improvement in therapeutic outcomes by optimizing platelet inhibition with prasugrel-Thrombolysis in Myocardial Infarction 38. Circulation 2008; 118: 1626-1636

23. Bae JP, Candrilli SD, Fortenberry $\mathrm{J}$ et al. Point-of-care platelet reactivity determination with VerifyNow-P2Y12 following administration of clopidogrel or prasugrel: data from a real-world, clinical care inpatient setting. Hosp Pract 2014; 42: 7-15.

24. Mayer K, Orban M, Bernlochner I et al. Predictors of antiplatelet response to prasugrel during maintenance treatment. Platelets 2015; 26: 53-58.

25. Akturk IF, Caglar FN, Erturk M et al. Hypertension as a risk factor for aspirin and clopidogrel resistance in patients with stable coronary artery disease. Clin Appl Thromb Hemost 2014; 20: 749-754

26. Al-Azzam SI, Alzoubi KH, Khabour OF et al. Factors that contribute to clopidogrel resistance in cardiovascular disease patients: environmental and genetic approach. Int J Clin Pharmacol Ther 2013; 51: 179-186

27. Verdoia M, Sartori C, Pergolini P et al. Prevalence and predictors of high-on treatment platelet reactivity with ticagrelor in ACS patients undergoing stent implantation. Vascul Pharmacol 2015; 6: 1537-1891 [Epub ahead of print]. 
28. Price MJ, Nayak KR, Barker CM et al. Predictors of heightened platelet reactivity despite dual-antiplatelet therapy in patients undergoing percutaneous coronary intervention. Am J Cardiol 2009; 103: 1339-1343.

29. Desai NR, Mega JL, Jiang S, Cannon CP, Sabatine MS. Interaction between cigarette smoking and clinical benefit of clopidogrel. J Am Coll Cardiol 2009; 53: 1273-1278.

30. Berger JS, Bhatt DL, Steinhubl SR et al; CHARISMA Investigators. Smoking, clopidogrel, and mortality in patients with established cardiovascular disease. Circulation 2009; 120: 2337-2344.

31. Yousef AM, Arafat T, Bulatova NR, Al-Zumyli R. Smoking behaviour modulates pharmacokinetics of orally administered clopidogrel. J Clin Pharm Ther 2008; 33: 439-449.

32. Gurbel PA, Bliden KP, Logan DK et al. The influence of smoking status on the pharmacokinetics and pharmacodynamics of clopidogrel and prasugrel: the PARADOX Study. J Am Coll Cardiol 2013; 62: 505-512.

33. Sofi F, Marcucci R, Gori AM et al. Clopidogrel non-responsiveness and risk of cardiovascular morbidity. An updated meta-analysis. Thromb Haemost 2010;103: 841-848

34. Montalescot G, Sideris G, Cohen R et al. Prasugrel compared with high-dose clopidogrel in acute coronary syndrome. The randomised double-blind ACAPULCO study. Thromb Haemost 2010; 103: 213-223.
35. Angiolillo DJ, Saucedo JF, Deraad R et al. Increased platelet inhibition after switching from maintenance clopidogrel to prasugrel in patients with acute coronary syndromes: results of the SWAP (SWitching Anti Platelet) study. J Am Coll Cardiol 2010; 56: 1017-1023.

36. Alexopoulos D, Xantopoulou I, Gkizas V et al. Randomized assessment of ticagrelor versus prasugrel antiplatelet effects in patients with ST elevation myocardial infarction. Circ Cardiovasc Interv 2012; 5: 797-804.

37. Laine $\mathrm{M}$, Glaubert $\mathrm{M}$, Frère $\mathrm{C}$ et al. Comparison of Platelet reactivity following prasugrel and cicagrelor loading dose in ST-Segment elevation Myocardial infarction patients: The COMPASSION study. Platelets 2014; 26: 570-572.

38. Alexopoulos D, Galati A, Xantopoulou I et al. Ticagrelor versus prasugrel in acute coronary syndrome patients with high on clopidogrel platelet reactivity following percutaneous coronary intervention. J Am Coll Cardiol 2012; 60: 193-199.

39. Kerneis M, Silvain J, Abtan J et al. Switching Acute coronary syndrome patients from prasugrel to clopidogrel. JACC: Cardiovascular interventions 2013; 6: 158-165.

40. Grdinic A, VojvodicD, Djukanovic N et al. PCl and clopidogrel: antiplatelet responsiveness and patient characteristics. Acta Cardiol 2011; 66: 333-340. 